\title{
Electrophysiological Correlates of Dream Recall During REM Sleep: Evidence from Multiple Awakenings and Within-Subjects Design
}

This article was published in the following Dove Press journal: Nature and Science of Sleep

\author{
Serena Scarpelli $\mathbb{D}^{1,2}$ \\ Chiara Bartolacci ${ }^{\prime}$ \\ Aurora D’Atri $\mathbb{D}^{1,3}$ \\ Milena Camaioni $\mathbb{D I}^{\prime}$ \\ Ludovica Annarumma (D) \\ Maurizio Gorgoni' \\ Chiara Cloos' \\ Michele Ferrara $\mathbb{( D}^{3}$ \\ Luigi De Gennaro (1D ${ }^{1,2}$ \\ 'Department of Psychology, Sapienza \\ University of Rome, Rome 00185, Italy; \\ ${ }^{2}$ IRCCS Fondazione Santa Lucia, Rome, \\ Italy; ${ }^{3}$ Department of Biotechnological \\ and Applied Clinical Sciences, University \\ of L'Aquila, Coppito, L'Aquila 67100, Italy
}

Correspondence: Luigi De Gennaro Department of Psychology, Sapienza University of Rome, Via Dei Marsi, 78, Rome 00185 , Italy

Tel +39-06-49917647

Fax +39-06-499177II

Email luigi.degennaro@uniromal.it
Purpose: In the current study, we aimed to investigate the EEG correlates of dream recall (DR) monitoring both the homeostatic and state-trait like factors. We assessed the influence of the time of night on the EEG correlates of DR from REM sleep. Specifically, we tested the continuity-hypothesis (on the theta EEG band) and the activation-hypothesis (on the delta and beta bands).

Methods: Twenty-seven subjects underwent polysomnography with multiple provoked awakenings during REM sleep. Only the subjects showing combinations of dream recall (REC) and non-REC (NREC) conditions in both first (1st-2nd sleep cycle) and second (3rd4th sleep cycle) part of the night were included in the analyses. The final sample was composed of 10 subjects (mean age $24 \pm 0.70$ ). EEG power spectra of the 5-min of REM sleep preceding each awakening were computed by a fast Fourier transform. The following frequency bands were considered: delta $(0.50-4.75 \mathrm{~Hz})$, theta $(5.00-7.75 \mathrm{~Hz})$, and beta $(16.00-24.75 \mathrm{~Hz})$. We also calculated the delta/beta power ratio as an integrated EEG index of activation.

Results: The $2 \times 2$ within-subjects ANOVA recall $\times$ time revealed: a) no significant effect for time and no interaction; b) significant differences over the occipital area in the beta band; c) significant differences over the parietal area for the activation index values. Overall, the results indicated that DR is associated with higher activation regardless of homeostatic pressure across the night of sleep.

Conclusion: In line with recent findings, we have shown that DR is predicted by desynchronized EEG activity during REM sleep, providing clear evidence in favor of the activationhypothesis. We have also confirmed that the EEG pattern of DR can be ascribed to state-like factors. Further studies should assess whether homeostatic modulation may interact with some dream features and the related EEG predictors.

Keywords: dream recall, REM sleep, EEG, activation hypothesis, multiple awakenings, sleep homeostasis

\section{Introduction}

Studies on neural correlates of dream experience suggested the potential influences of circadian, ultradian, and homeostatic factors on dream features. ${ }^{1}$ Most investigations neglected the impact of these variables on EEG predictors of dream recall $(\mathrm{DR})^{2-8}$ and very few studies provided information about this issue.

Chellappa et $\mathrm{al}^{9}$ assessed the EEG correlates of DR by using a 40-h multiple nap protocol. They found that the relationship between DR and specific EEG patterns is modulated by the level of melatonin concentration detected in the subjects' saliva 
samples before naps. The authors reported that DR was associated with less frontal delta and centro-parietal spindles during NREM sleep. Moreover, during REM sleep, DR was linked to lower alpha and beta power in the frontal areas and higher power in the occipital region. The link between DR and these specific bands during REM and NREM sleep could be ascribed to the repeated offset and re-onset of sleep. In other words, this finding suggests that altered ultradian and circadian rhythms affect mental sleep activity, ${ }^{1}$ and, hence, the EEG predictors of DR.

More recently, a study using the Multiple Sleep Latency Test (MSLT) of narcoleptic patients showed no effects of the circadian phase on the EEG correlates of dream experience. DR was predicted by higher EEG desynchronization in both NREM and REM sleep. ${ }^{10}$ Consistently, a within-subject nap protocol on healthy subjects found that DR from stage 2 NREM sleep was predicted by lower delta activity. The authors controlled the influence of the sleep cycle on DR, revealing no interactions for any derivation and frequency range. ${ }^{11}$

Besides, Zhang and coworkers ${ }^{12}$ reported that reduced slow oscillations were related to dreaming experience during stage 2 NREM sleep. Nevertheless, at sleep onset, this association showed the opposite direction at sleep onset: DR was linked to increased slow EEG activity.

The available literature partly is consistent with the activation-hypothesis, supporting the notion of a relationship between dream experience and an electrophysiological milieu characterized by higher arousal. ${ }^{13}$ However, no definitive conclusion can be drawn since the information is scarce and stems from different experimental protocols, ie, nap, MSLT, or night recordings.

The so-called "state-/trait-like" issue should also be taken into account. ${ }^{14}$ Indeed, several studies point out that the EEG correlates of DR depend on the neurophysiological scenario characterizing the sleep period immediately before the dream collection (ie, the state-like hypothesis). ${ }^{6,10}$ Some findings from within-subjects designs confirmed the link between higher activation and dream experience. ${ }^{10,11}$ Also, Scarpelli et al $^{6}$ highlighted the involvement of frontal theta oscillations during REM sleep in predicting DR, supporting the idea that cognitive processes during sleep and wakefulness share similar mechanisms. ${ }^{14,15}$ However, it should be noted that specific stable differences characterizing individuals could impact on dream features (ie, the trait-like hypothesis). In this respect, neuroimaging studies revealed that volumetric and structural measures of brain networks, such as the amygdala and hippocampus, were related to specific qualitative characteristics of dream reports. ${ }^{16,17}$ Moreover, Eichenlaub et $\mathrm{al}^{18}$ observed that the activation of the temporo-parietal-junction and the medial prefrontal cortex was associated with higher DR frequency.

Overall, successful and unsuccessful DR differs in a very topographic-specific manner. Starting from the knowledge that the distribution/amount of slow-wave activity decreases across the night, ${ }^{19}$ we cannot rule out that EEG patterns predicting DR may be affected by the homeostatic factors. To our knowledge, no studies investigated the EEG correlates of DR monitoring both the state-trait like factors and homeostatic variables. In the current study, we tested the influence of the time of night (homeostatic factor) on the EEG correlates of DR from REM sleep, considering two hypotheses:

1) Activation-hypothesis: ${ }^{13}$ higher beta and lower delta power promote DR from REM sleep;

2) Continuity-hypothesis: ${ }^{2,20}$ higher frontal theta oscillations promote DR from REM sleep.

Specifically, we aimed to use a multiple awakenings protocol on healthy subjects, and the predictive power of EEG oscillations for DR was assessed by comparing within-subjects couples of REM sleep intervals followed by the recall (REC) or non-recall (NREC) during the first and second part of the night.

\section{Materials and Methods}

\section{Subjects}

Twenty-seven healthy volunteers $[11 \mathrm{~F}$; mean age \pm standard error (SE) $24.16 \pm 0.16$ ] were enrolled to participate in the current study. All subjects were students recruited from the "Sapienza" University of Rome. Participants met the following inclusion criteria: no chronic illness, neurological or mental disorders, no assumption of medications, no alcohol or drug addiction, regular sleep habits (24.00-$8.00 \pm 1 \mathrm{~h}$ ), absence of excessive daytime sleepiness, no other sleep disorders. A semi-structured interview and the Pittsburgh Sleep Quality Index (PSQI ${ }^{21}$ ) was administered at the recruitment stage.

All subjects undergo two-night recordings. However, according to the aims of the study, we included in the final sample only subjects who reported at least one REC and one NREC condition both during the first (T1: 1st-2nd sleep cycle) and the second (T2: 3rd-4th sleep cycle) part of the night. In other words, we aimed to achieve - for each 
subject - the combinations REC-NREC during T1 and RECNREC during T2 to carry out analyses on intraindividual measures. Hence, among the 27 participants: 10 participants obtained the required REC-NREC coupling during T1, and 19 participants obtained the required REC-NREC coupling in T2. Overall, the final sample was composed of 10 subjects reporting REC-NREC couplings in both T1 and T2 $(5 \mathrm{~F}$; mean age \pm SE $24 \pm 0.70$ ) for a total of 20 awakenings from REM sleep stage (see, Figure 1).

The study was carried out in accordance with the recommendations of the Declaration of Helsinki. The Institutional Review Board of the Department of Psychology of the "Sapienza" University of Rome approved the experimental protocol.

\section{Procedure}

All participants signed informed consent for the collection and treatment of data prior to participating to the experiment. Each subject participated in two experimental sessions structured as follows: a) EEG montage; b) subjects were instructed to consider any distinct mental activity occurring during sleep as a dream experience; c) polysomnography (PSG) with planned-provoked awakenings; d) final morning awakening.
In order to ensure comparability between the two experimental nights, each subject was required to keep regular sleep-wake schedule during the week preceding the experimental session. The compliance was verified by a sleep diary. Experimental sessions were separated by at least one week. Subjects were also requested to abstain from caffeine and alcohol during the day of experimental session. The timing of the sleep-wake schedule during the protocol was adjusted to individual sleep-wake habits.

Awakenings were planned for each sleep cycle from REM sleep stage after at least 5 min without stage shifts by calling out the first name of the subject through an interphone system. Participants were asked to report if they had (REC condition) or had not (NREC condition) a dream experience just before awakening. We did not collect dream contents to avoid an excessive activation that can interfere with subsequent sleep.

\section{Polysomnographic Recording}

The PSG was recorded in a sound-proof, temperaturecontrolled room inside the sleep laboratory. A Brain Amp MR plus system (Brain Products GmbH, Gilching, Munich) was used to record EEG throughout the nights at a sampling rate of $250 \mathrm{~Hz}$. The 28 unipolar EEG
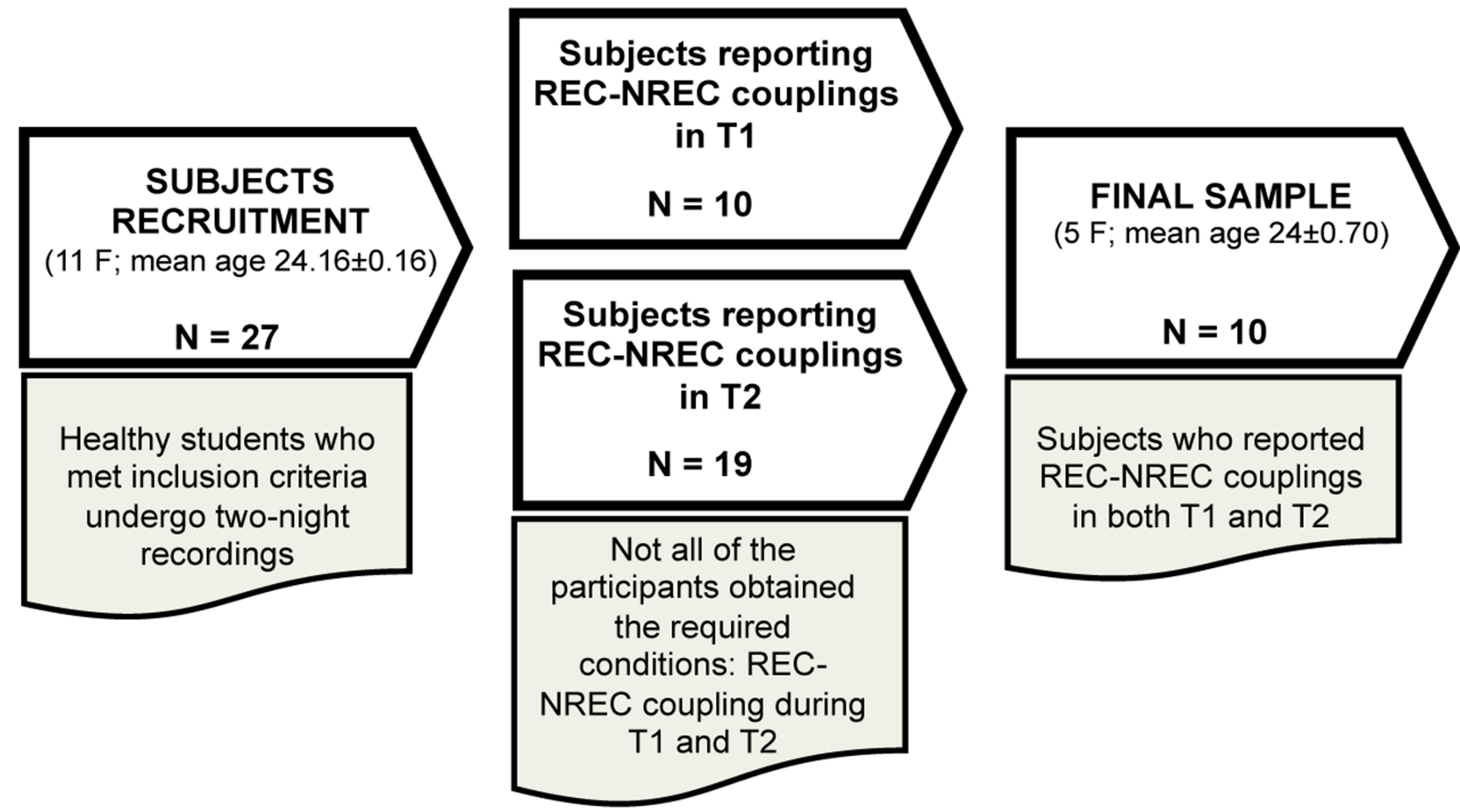

Figure I Flowchart of the final sample selection procedure. REC, recall; NREC, non-recall; TI, first part of the night (Ist -2 nd sleep cycle); T2, second part of the night (3rd -4 th sleep cycle). 
derivations placed following the international 10-10 system (C3, C4, Cp1, Cp2, Cp5, Cp6, Cz, F3, F4, F7, F8, Fc1, Fc2, Fc5, Fc6, Fp1, Fp2, Fz, O1, O2, Oz, P3, P4, P7, $\mathrm{P} 8, \mathrm{Pz}, \mathrm{T} 7, \mathrm{~T} 8$ ) were recorded by using an elastic cap (Easycap, Falk Minow, Munich) with sintered $\mathrm{Ag}-\mathrm{AgCl}$ electrodes. The cortical EEG signals were referenced online to the averaged mastoids (A1, A2) and the ground electrode was located anterior to $\mathrm{Fz}(\mathrm{Fpz})$. Electrooculogram (EOG) and submental electromyogram (EMG) channels were also recorded. EEG signals were analogically high-pass filtered with the time constant of $0.3 \mathrm{~s}$ and lowpass filtered at $30 \mathrm{~Hz}$. A notch filter was applied $(50 \mathrm{~Hz})$. Electrode impedance was fixed below $5 \mathrm{k} \Omega$.

\section{Data Analysis}

All analyses were performed only on the data collected from the 10 subjects who reported the combination of REC and NREC conditions in both first (T1) and second (T2) part of the night.

\section{Sleep Architecture}

PSG data were scored considering $20 \mathrm{~s}$ epochs according to the Rechtschaffen and Kales criteria. ${ }^{22}$ SWS scoring rigidly fulfilled the $>75-\mu \mathrm{V}$ amplitude criterion. The traditional sleep parameters were calculated for each night recording: sleep stages latency and duration, total bed time (TBT), total sleep time (TST, ie, the sum of time spent in stage 1, stage 2, SWS and REM), wake after sleep onset (WASO), number of awakenings and sleep efficiency index (SEI $=$ TST/TBT x 100).

\section{Quantitative EEG Analysis}

The EEG signals of the 5 min of REM sleep preceding each awakening were visually inspected for $8 \mathrm{~s}$ epochs to reject ocular and muscular artifacts. Power spectra for the 28 scalp derivations in the $0.5-29 \mathrm{~Hz}$ range were computed by a Fast Fourier transform (FFT) routine on consecutive $4 \mathrm{~s}$ artefact-free epochs, resulting in a frequency resolution of $0.25 \mathrm{~Hz}$. The values of EEG power from adjacent frequency bins for each scalp derivation expressed as percentage of the total power spectrum within the whole topography - were summed together to obtain the frequency bands considered to test the hypothesis of the current study: delta $(0.50-4.75 \mathrm{~Hz})$, theta $(5.00-7.75 \mathrm{~Hz})$, and beta $(16.00-24.75 \mathrm{~Hz})$.

\section{Statistical Analysis}

All analyses were performed using the software package MATLAB (The Math Works, Inc., MA, USA) and its statistics toolbox.

Firstly, to ascertain the comparability between the two nights recorded for each subject, we performed statistical comparisons (paired $t$-test) between the PSG measures of night 1 (N1) and night 2 (N2). To adjust the $\alpha$-value for multiple comparisons, Bonferroni correction was applied. ${ }^{23,24}$ As suggested by Sankoah et al, ${ }^{24}$ we calculated the mean correlation between the dependent variables $(r=0.0319)$ and the $\alpha$ level was adjusted to 0.0042 .

In order to understand whether the awakenings from 1st or 2nd part of the night affect the EEG activity prior to the subsequent DR, we compared the relative EEG power spectra by means of two-way within design ANOVAs recall $(\mathrm{REC}$ vs NREC) $\times$ time $(\mathrm{T} 1$ vs $\mathrm{T} 2)$, performed separately for each scalp derivation and frequency band (delta, theta, beta). Bonferroni correction was applied. ${ }^{23,24}$ The mean correlation between the dependent variables is $\mathrm{r}=0.3510$, and the $\alpha$ level was adjusted to 0.0028 .

Moreover, in order to directly test the activationhypothesis, we also calculated the delta/beta power ratio as an integrated EEG index of activation. Previously, this index was proved to be reliable predictor of dream recall both in healthy and clinical samples. ${ }^{8,10}$ The $2 \times 2$ ANOVA recall $\times$ time was carried out also for the delta/beta power ratio values, for each scalp derivations. To adjust the $\alpha$ level Bonferroni correction was applied. ${ }^{23,24}$ Considering the mean correlation between the dependent variables $(r=0.5322)$, $\alpha$ level was adjusted to 0.011 .

\section{Results}

\section{Sleep Architecture}

Table 1 reports the results of the paired t-tests comparing N1 and N2 sleep measures. Stage 1 latency, stage 3 latency and SEI showed slight differences between the two nights coherent with the first-night effect ${ }^{25}$ However, no difference survived after the Bonferroni correction $(\alpha=0.0042)$.

\section{High-Frequency Activity Predicts Dream Recall Regardless of Homeostatic Pressure}

Figure 2 depicts the topographic distribution of mean relative EEG power in the delta, theta and beta frequency bands for the 5-min of REM sleep before each awakening 
Table I Sleep Macrostructure

\begin{tabular}{|l|l|l|l|l|l|l|}
\hline \multicolumn{2}{|l}{} & \multicolumn{2}{l}{ NI } & N2 & \multirow{2}{*}{ t-values } \\
\cline { 2 - 5 } & Mean & ES & Mean & ES & \\
\hline Stage I latency (min) & 17.20 & 5.11 & 7.70 & 2.70 & 2.341 & 0.044 \\
Stage 2 latency (min) & 18.37 & 5.19 & 9.47 & 2.91 & 2.239 & 0.052 \\
Stage 3 latency (min) & 81.97 & 14.78 & 35.40 & 6.34 & 2.808 & 0.020 \\
REM sleep latency (min) & 134.10 & 13.82 & 110.33 & 12.45 & 1.499 & 0.168 \\
Stage I (min) & 12.26 & 2.18 & 10.93 & 1.20 & 0.859 & 0.412 \\
Stage 2 (min) & 253.59 & 22.78 & 253.30 & 22.34 & 0.013 & 0.990 \\
SWS (min) & 28.11 & 5.82 & 36.13 & 5.33 & -1.421 & 0.189 \\
REM (min) & 54.97 & 1.80 & 50.70 & 4.63 & 1.035 & 0.328 \\
WASO (min) & 67.20 & 18.05 & 38.67 & 4.90 & 2.065 & 0.065 \\
Awakenings (\#) & 23.80 & 1.44 & 23.60 & 2.13 & 0.115 & 0.911 \\
TST (min) & 348.93 & 19.56 & 351.07 & 30.67 & -0.123 & 0.905 \\
TBT (min) & 433.73 & 11.88 & 399.77 & 35.40 & 2.486 & 0.035 \\
SEl \% (TST/TBT) & 80.59 & 0.04 & 87.85 & 0.08 & -2.311 \\
\hline
\end{tabular}

Notes: Means and standard errors (SEs) of the PSG variables of the Night I (NI) and Night 2 (N2). The results of the paired $t$-test and p-values are also reported. No difference survived after the Bonferroni correction $(\alpha=0.0042)$.

Abbreviations: SWS, slow wave activity; REM, rapid eye movement; WASO, wake after sleep onset; TST, total sleep time; TBT, total bed time; SEI, sleep efficiency index.

of the REC and NREC conditions during T1 and T2. It should be noted that EEG regional patterns are substantially preserved in both REC and NREC conditions during the 1st (T1) and 2nd (T2) part of the night.

Figure 3 depicts the topographic maps of the $F$ coefficients from the two-way ANOVAs "recall $\times$ time" performed for each band and derivation. The main effect of recall was observed. Significant differences were found over occipital area in the beta band: O1 $(\mathrm{F}=19.69$; $\mathrm{p}=0.0016)$ and $\mathrm{O} 2(\mathrm{~F}=21.21 ; \mathrm{p}=0.0013)$.

No significant effect was revealed for Time and no interaction was found. Overall, the beta activity was associated to the presence of DR regardless of homeostatic pressure across the night of sleep.

\section{The Delta/Beta Ratio Predicts Dream Recall}

Figure 4 shows the topographic distribution of the activation index values (delta/beta) in REC and NREC conditions during $\mathrm{T} 1$ and $\mathrm{T} 2$ and $\mathrm{F}$ coefficients from the two-way ANOVAs "recall $\times$ time" performed on the activation index. No significant effect was revealed for Time and no interaction was found. The main effect of recall was again significant. In particular, significant differences were found over parietal area: $\mathrm{P} 4(\mathrm{~F}=11.44 ; \mathrm{p}=0.0081)$.

These findings showed that higher level of cortical activation (ie, reduced values of the activation index) during the last 5-min of REM sleep before awakening in the parietal region predicts DR.

\section{Discussion}

As far as we know, this is the first challenging study that investigated the EEG correlates of DR taking under control homeostatic pressure (time of the night) during the night in a within-subjects design. We showed that the occipital beta power is linked to DR regardless of the time of the night. More directly, the activation index expressed as the delta/beta ratio confirmed that higher level of cortical arousal during REM sleep predicts the subsequent DR. The results substantially replicate previous studies indicating the association between desynchronized EEG activity and dream experience during REM sleep. ${ }^{5,10}$ Moreover, we showed that homeostatic pressure does not interact with EEG predictors of DR from REM sleep over the night.

Our findings on EEG correlates of DR during REM sleep overlap the EEG pattern observed during NREM sleep, ${ }^{5,8,10,11}$ providing support to the one generator model of mental sleep activity. ${ }^{26}$ In this vein, several studies revealed the relationship between higher cortical arousal during NREM sleep (increased fast-frequencies and/or reduced slow-frequencies) and DR both in healthy and clinical groups. ${ }^{5,8,9,11,12}$

Besides, we confirmed that the parietal and occipital regions are involved in sleep mentation, as suggested by early studies. ${ }^{27}$ Consistently, Eichenlaub and collaborators ${ }^{18}$ 


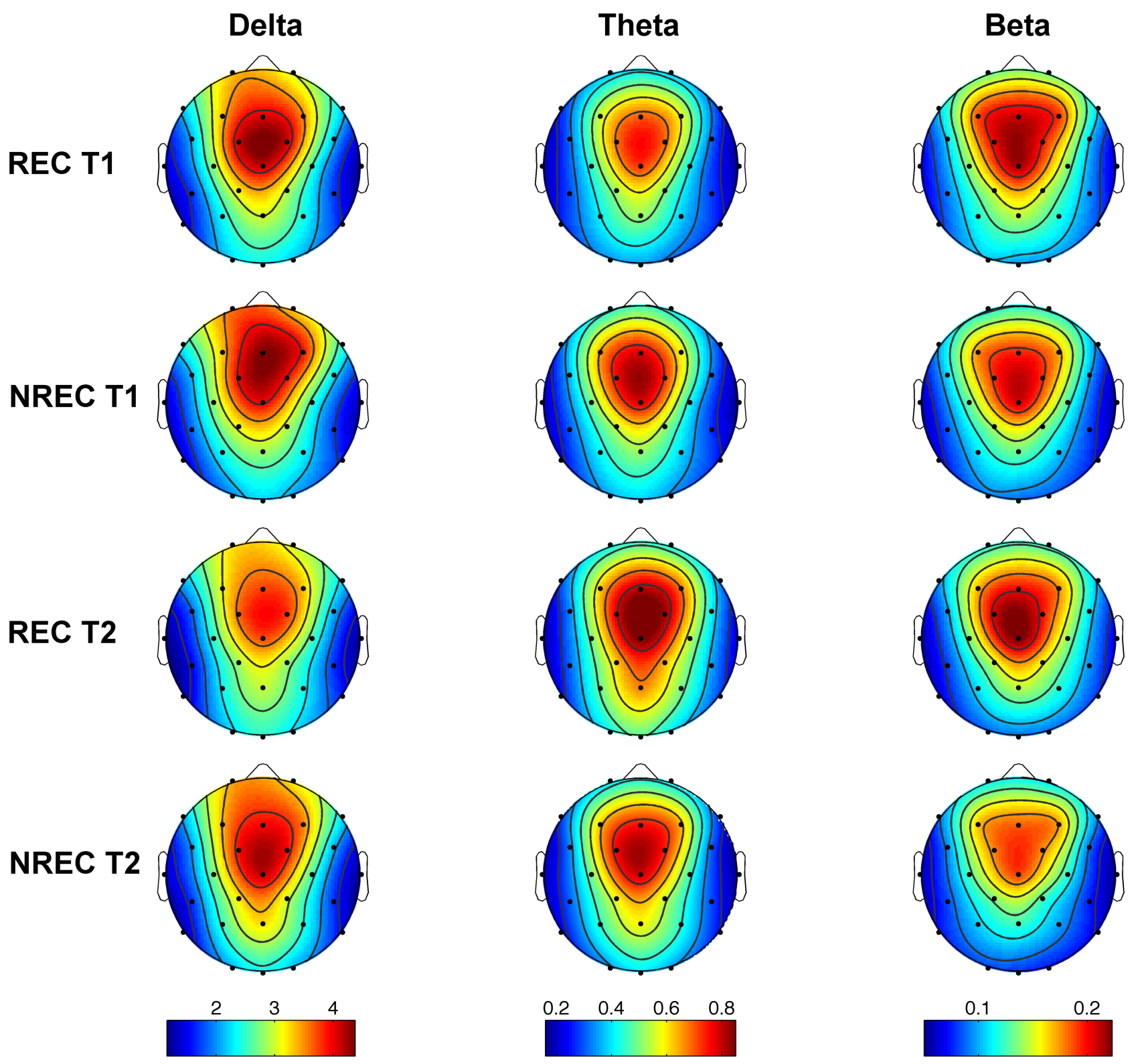

Figure 2 Topographic distribution of EEG powers during the last 5-min of rem sleep preceding awakenings. The EEG activity for the delta $(0.5-4.75 \mathrm{~Hz})$, theta $(5-7.75 \mathrm{~Hz})$ and beta $(16-24.75 \mathrm{~Hz})$ frequency bands is reported, expressed as percentage of the total EEG power within the whole topography. The maps are scaled between the minimal and maximal power values for each frequency band considering the recall (REC) condition during TI (Ist row) and T2 (3rd row), and non-recall (NREC) condition during TI (2nd row) and T2 (4th row). The maps are based on 28 derivations (electrode positions indicated by black dots) with averaged mastoid reference. Values are color-coded and plotted at the corresponding position on the planar projection of the hemispheric scalp model. Values between electrodes were interpolated (biharmonic spline interpolation).

revealed that high dream recall frequency was associated with increased activity in the temporo-parietal junction at rest. In particular, the results replicated the idea that the so-called "posterior hot zone" of the brain is responsible for dreaming. ${ }^{5,28}$ Indeed, a recent study using EEG high-density recordings and serial provoked awakenings ${ }^{5}$ showed that dream experience is related with locally decreasing of slowwave activity in the posterior zone including medial and lateral occipital lobe, extending to the precuneus and posterior cingulate gyrus. ${ }^{28-30}$ Also, faster frequencies than $25 \mathrm{~Hz}$ (ie, the gamma activity) over temporal and frontal regions during REM sleep were associated with DR. ${ }^{5}$ However, some specific content related to perceiving dimension correlated with the posterior regions (ie, parietal, occipital, and temporal). For instance, dream reports containing spatial settings were associated with increased high-frequency EEG activity in the right posterior cortex. ${ }^{5}$ Moreover, the crucial role of desynchronized EEG activity in consciousness experience was highlighted by 


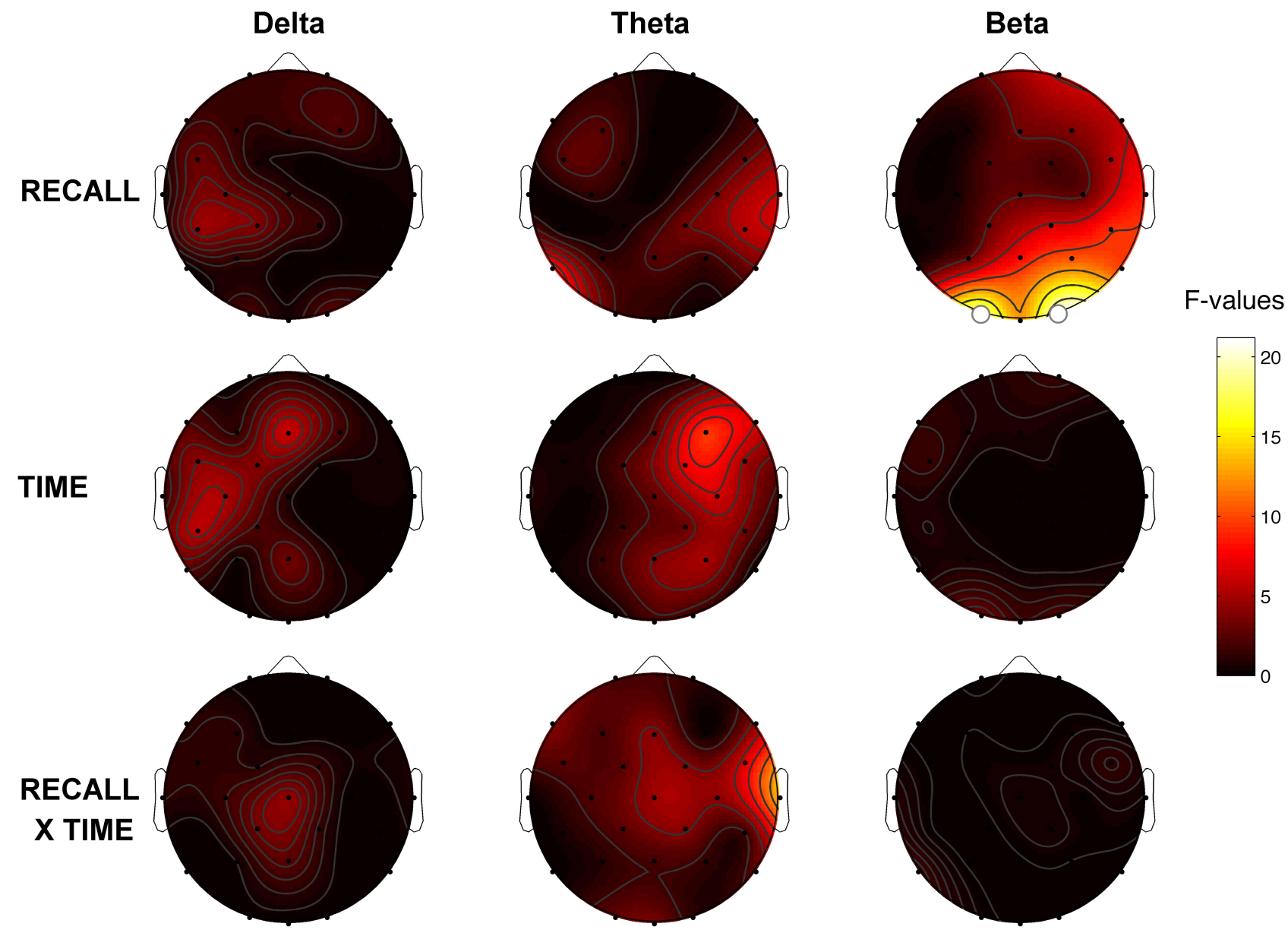

Figure 3 Statistical comparisons on EEG powers during the last 5-min of rem sleep preceding awakenings. Statistical maps reporting the F-values of two ways ANOVAs recall $\times$ time for delta, theta and beta bands. The main effects (recall; time) are reported in the first two rows and the interactions (recall $\times$ time) are depicted in the $3 r d$ row. The maps are based on 28 derivations (electrode positions indicated by black dots) with averaged mastoid reference. Values are color-coded and plotted at the corresponding position on the planar projection of the hemispheric scalp model. Values between electrodes were interpolated (biharmonic spline interpolation). Significant effect of the recall factor was found (OI: $F=19.69, p=0.0016 ; O 2$ : $F=21.21, p=0.0013)$. White dots indicate significant effects after the Bonferroni correction $(\alpha=0.0028)$.

some findings on lucid dreams revealing that increased beta activity over parietal regions ${ }^{31}$ or gamma band in the frontal region $^{32}$ are related with the awareness to have a dream.

Contrary to the initial continuity-hypothesis, we did not observe any differences between REC and NREC conditions in the theta range, recognized as crucial in the retrieving episodic memory and dream contents. ${ }^{2,6,7,33}$ Notwithstanding, we have to underline that continuityand activation-hypothesis are not mutually exclusive. Actually, the local activation of the posterior cerebral cortex is a consolidated marker of consciousness and visual experiences also during wakefulness. ${ }^{28}$ Once again, our findings suggest that - to some extent - cognitive processes during waking state and sleep shared similar mechanisms.

Concerning the lack of effect for the factor Time (ie, the comparison between the first and last half of the night), it may be partly explained by smaller homeostatic effects involving the posterior areas. ${ }^{34}$ In other words, the posterior areas affected by the differences associated to dream recall also are those showing the smallest differences in the homeostatic pressure.

Finally, the within-subjects design allows us to posit that the neural correlates of dream experience depend on the electrophysiological scenario during the sleep period before the awakening. ${ }^{14}$

\section{Limitations}

We are aware that our protocol suffers from some limitations that should be addressed in future studies. Firstly, the current study did not investigate oneiric contents. On the one side, this methodological choice allowed to promote an easy reonset after provoked awakening. On the other side, we could not analyze any relationship between qualitative or 
A

REC T1

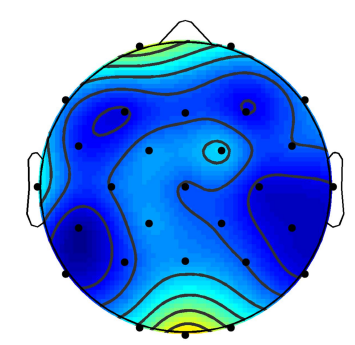

NREC T1

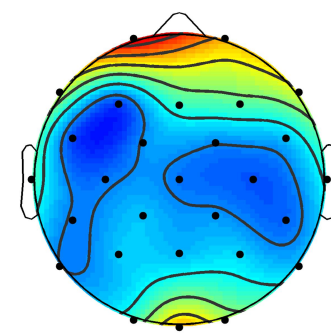

REC T2

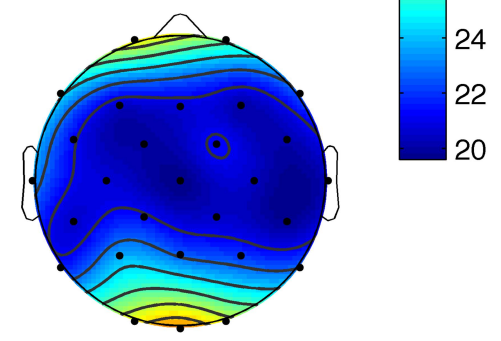

NREC T2

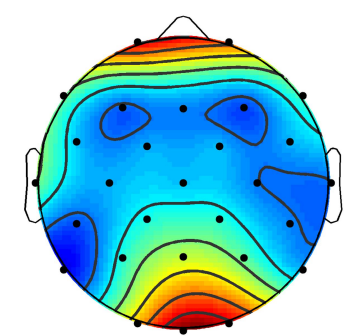

B

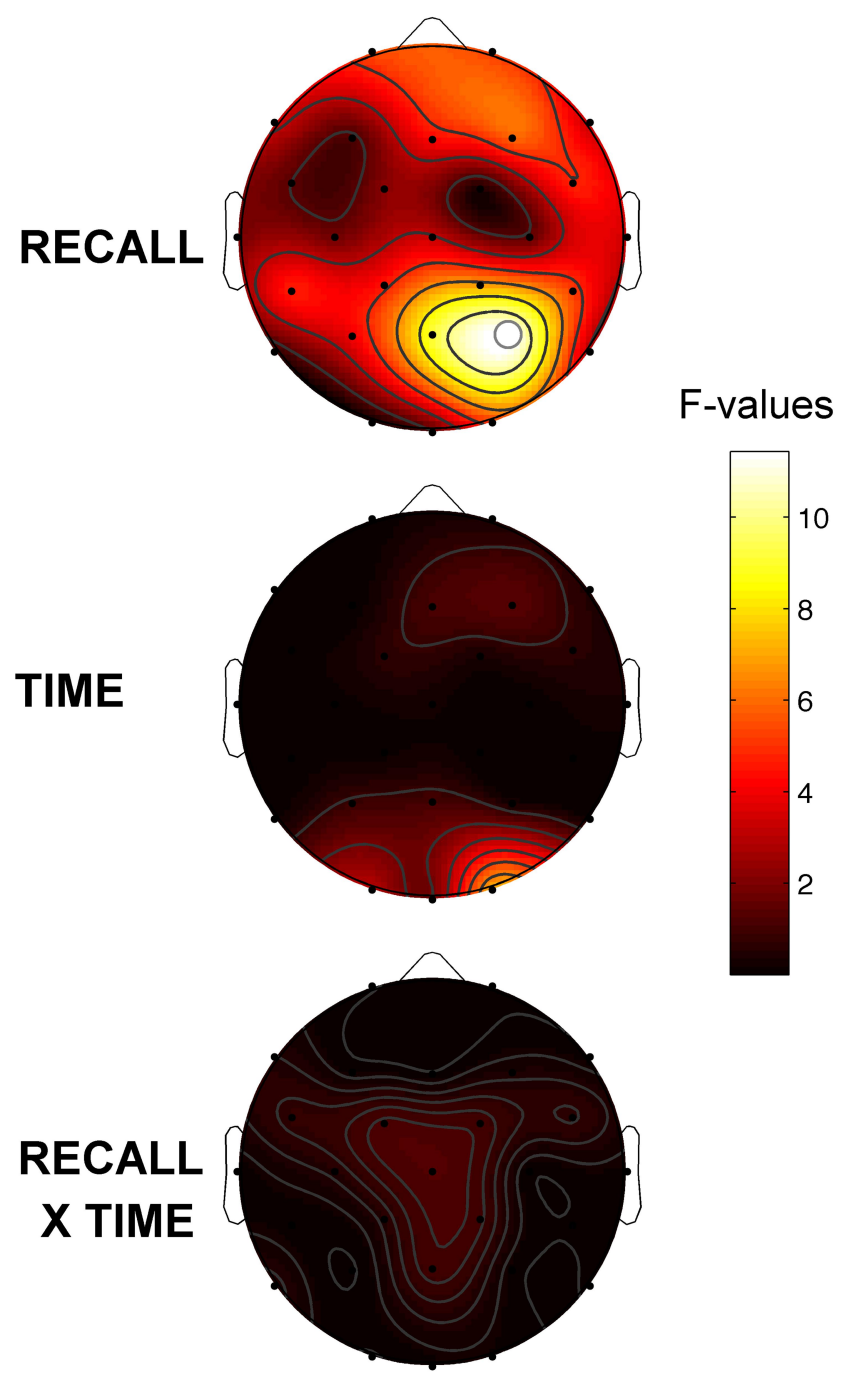

Figure 4 Topographic distribution and statistical maps of the activation index values. Panel A reports the topographic distribution of the activation index values (delta/beta ratio) of the last 5-min of REM sleep preceding awakenings of recall (REC) during TI (Ist row) and T2 (3rd row) and non-recall (NREC) during TI (2nd row) and T2 (4th row). Panel B depicts statistical maps reporting the F-values of two ways ANOVAs recall $\times$ time for activation index values. The main effects (recall; time) are reported in the first two rows and the interactions (recall $\times$ time) are depicted in the 3rd row. The maps are based on 28 derivations (electrode positions indicated by black dots) with averaged mastoid reference. Values are color-coded and plotted at the corresponding position on the planar projection of the hemispheric scalp model. Values between electrodes were interpolated (biharmonic spline interpolation). Significant effect of the recall factor was found (P4: $\mathrm{F}=\mathrm{II} .44, \mathrm{p}=0.008 \mathrm{I})$. White dots indicate significant effects after the Bonferroni correction $(\alpha=0.01 \mathrm{I})$.

quantitative dream features and sleep EEG patterns. Besides, our sample is totally composed of young university students and this could represent a bias that does not allow us to generalize our results. For instance, since the literature on dreaming during lifespan is very poor, ${ }^{7,83}$ within-subject studies will carry out on larger age ranges (eg, older adults; children). Although the difficulty of obtaining the NREC condition during REM sleep is well known, ${ }^{1}$ our sample consisting of 10 subjects with within-subject comparisons is relatively small and further investigation should enlarge the sample size to strengthen the current findings.

\section{Conclusion}

In keeping with recent studies, ${ }^{5,9,10}$ we have shown that DR during REM sleep is predicted by desynchronized EEG activity, expressed by higher beta power over the occipital area and the activation index values over the right parietal location. In other words, we provided clear evidence in favor of the activation-hypothesis. We have also confirmed that the EEG pattern of DR can be ascribed to state-like factors.

Finally, the current investigation, for the first time, highlighted that the EEG correlates of DR upon awakenings from 
REM sleep are not affected by the time of the night. However, considering that dream length and quality seem to change across the night, ${ }^{36}$ future studies should assess whether homeostatic pressure modulation may interact with some dream features and the related EEG predictors. ${ }^{1,36}$

\section{Disclosure}

The authors report no conflicts of interest in this work.

\section{References}

1. Nielsen TA. Chronobiological features of dream production. Sleep Med Rev. 2004;8(5):403-424. doi:10.1016/j.smrv.2004.06.005

2. Marzano C, Ferrara M, Mauro F, et al. Recalling and forgetting dreams: theta and alpha oscillations during sleep predict subsequent dream recall. $J$ Neurosci. 2011;31(18):6674-6683. doi:10.1523/ JNEUROSCI.0412-11.2011

3. Esposito MJ, Nielsen TA, Paquette T. Reduced Alpha power associated with the recall of mentation from Stage 2 and Stage REM sleep. Psychophysiology. 2004;41(2):288-297. doi:10.1111/j.14698986.00143.x

4. Takeuchi T, Ogilvie RD, Murphy TI, Ferrelli AV. EEG activities during elicited sleep onset REM and NREM periods reflect different mechanisms of dream generation. Electroencephalograms. Rapid eye movement. Clin Neurophysiol. 2003;114(2):210-220. doi:10.1016/ S1388-2457(02)00385-1

5. Siclari F, Baird B, Perogamvros L, et al. The neural correlates of dreaming. Nat Neurosci. 2017;20(6):872-878. doi:10.1038/nn.4545

6. Scarpelli S, Marzano C. D'Atri A, Gorgoni M, Ferrara M, De Gennaro L. State-or trait-like individual differences in dream recall: preliminary findings from a within-subjects study of multiple nap REM sleep awakenings. Front Psychol. 2015;6:928. doi:10.3389/ fpsyg.2015.00928

7. Scarpelli S, D'Atri A, Bartolacci C, Mangiaruga A, Gorgoni M, De Gennaro L. Oscillatory EEG Activity During REM Sleep in Elderly People Predicts Subsequent Dream Recall After Awakenings. Front Neurol. 2019;10:985. doi:10.3389/fneur.2019.00985

8. Scarpelli S, D'atri A, Bartolacci C, et al. Dream recall upon awakening from non-rapid eye movement sleep in older adults: electrophysiological pattern and qualitative features. Brain Sci. 2020;10:343.

9. Chellappa SL, Frey S, Knoblauch V, Cajochen C. Cortical activation patterns herald successful dream recall after NREM and REM sleep. Biol Psychol. 2011;87(2):251-256. doi:10.1016/j.biopsycho.2011.03.004

10. D'Atri A, Scarpelli S, Schiappa C, et al. Cortical activation during sleep predicts dream experience in narcolepsy. Ann Clin Transl Neurol. 2019;6(3):445-455. doi:10.1002/acn3.718

11. Scarpelli S, D'Atri A, Mangiaruga A, et al. Predicting Dream Recall: EEG Activation During NREM Sleep or Shared Mechanisms with Wakefulness? Brain Topogr. 2017;30(5):629-638. doi:10.1007/ s10548-017-0563-1

12. Zhang J, Wamsley EJ. EEG predictors of dreaming outside of REM sleep. Psychophysiology. 2019;56(7):e13368. doi:10.1111/psyp. 13368

13. Antrobus J, Kondo T, Reinsel R, Fein G. Dreaming in the late morning: summation of rem and diurnal cortical activation. Conscious Cogn. 1995;4:275-299. doi:10.1006/ccog.1995.1039

14. Scarpelli S, D’Atri A, Gorgoni M, Ferrara M, De Gennaro L. EEG oscillations during sleep and dream recall: state- or trait-like individual differences? Front Psychol. 2015;6:605.

15. Cipolli C, Ferrara M, De Gennaro L, Plazzi G. Beyond the neuropsychology of dreaming: insights into the neural basis of dreaming with new techniques of sleep recording and analysis. Sleep Med Rev. 2017;35:8-20.
16. De Gennaro L, Cipolli C, Cherubini A, et al. Amygdala and hippocampus volumetry and diffusivity in relation to dreaming. Hum Brain Mapp. 2011;32(9):1458-1470. doi:10.1002/hbm.21120

17. De Gennaro L, Lanteri O, Piras F, et al. Dopaminergic system and dream recall: an MRI study in Parkinson's disease patients. Hum Brain Mapp. 2016;37(3):1136-1147. doi:10.1002/hbm.23095

18. Eichenlaub J-B, Nicolas A, Daltrozzo J, Redoute J, Costes N, Ruby P. Resting brain activity varies with dream recall frequency between subjects. Neuropsychopharmacology. 2014;39(7):1594-1602. doi:10. 1038/npp.2014.6

19. Borbély AA, Achermann P. Sleep Homeostasis and Models of Sleep Regulation. J Biol Rhythms. 1999.

20. Schredl M, Hofmann F. Continuity between waking activities and dream activities. Conscious Cogn. 2003;12:298-308. doi:10.1016/ S1053-8100(02)00072-7

21. Curcio G, Tempesta D, Scarlata S, et al. Validity of the Italian Version of the Pittsburgh Sleep Quality Index (PSQI). Neurol Sci. 2013;34(4):511-519. doi:10.1007/s10072-012-1085-y

22. Rechtschaffen A, Kales A. A Manual of Standardized Terminology, Techniques and Scoring System for Sleep Stages of Human Subjects. Los Angeles: UCLA Brain Information Service; 1978.

23. Perneger TV. What's wrong with Bonferroni adjustments. BMJ. 1998;316:1236-1238. doi:10.1136/bmj.316.7139.1236

24. Sankoh AJ, Huque MF, Dubey SD. Some comments on frequently used multiple endpoint adjustment methods in clinical trials. Stat Med. 1997;16(22):2529-2542. doi:10.1002/(SICI)1097-0258(19971130) 16:22<2529::AID-SIM692>3.0.CO;2-J

25. Curcio G, Ferrara M, Piergianni A, Fratello F, De Gennaro L. Paradoxes of the first-night effect: a quantitative analysis of antero-posterior EEG topography. Clin Neurophysiol. 2004;115 (5):1178-1188. doi:10.1016/j.clinph.2003.12.018

26. Scarpelli S, Gorgoni M, D'Atri A, Ferrara M, De Gennaro L. Structural and Functional Differences in Brain Mechanisms of Dream Recall. Handbook of Behavioral Neuroscience. 2019.

27. Solms M. Neurobiology and the Neurological Basis of Dreaming. 2011.

28. Koch C, Massimini M, Boly M, Tononi G. Neural correlates of consciousness: progress and problems. Nat Rev Neurosci. 2016;17:307-321.

29. Boly M, Massimini M, Tsuchiya N, Postle BR, Koch C, Tononi G. Are the neural correlates of consciousness in the front or in the back of the cerebral cortex? Clinical and neuroimaging evidence. J Neurosci. 2017;37(40):9603-9613. doi:10.1523/JNEUROSCI.3218-16.2017

30. Storm JF, Boly M, Casali AG, et al. Consciousness regained: disentangling mechanisms, brain systems, and behavioral responses. J Neurosci. 2017;37(45):10882-10893. doi:10.1523/JNEUROSCI. 1838-17.2017

31. Holzinger B, LaBerge S, Levitan L. Psychophysiological correlates of lucid dreaming. Dreaming. 2006;16(2):88-95. doi:10.1037/10530797.16.2.88

32. Voss U, Holzmann R, Hobson A, et al. Induction of self awareness in dreams through frontal low current stimulation of gamma activity. Nat Neurosci. 2014;17(6):810-812. doi:10.1038/nn.3719

33. Eichenlaub J-B, van Rijn E, Gaskell MG, et al. Incorporation of recent waking-life experiences in dreams correlates with frontal theta activity in REM sleep. Soc Cogn Affect Neurosci. 2018;13 (6):637-647. doi:10.1093/scan/nsy041

34. Marzano C, Ferrara M, Curcio G, Gennaro LD. The effects of sleep deprivation in humans: topographical electroencephalogram changes in non-rapid eye movement (NREM) sleep versus REM sleep. J Sleep Res. 2010;19(2):260-268. doi:10.1111/j.1365-2869.2009.00776.x

35. Scarpelli S, Bartolacci C, D’Atri A, Gorgoni M, De Gennaro L. Mental Sleep Activity and Disturbing Dreams in the Lifespan. Int J Environ Res Public Health. 2019;16(19):3658. doi:10.3390/ ijerph 16193658

36. Nielsen T. Ultradian, Circadian, and Sleep-Dependent Features of Dreaming. In: Principles and Practice of Sleep Medicine. Fifth:2010. 


\section{Publish your work in this journal}

Nature and Science of Sleep is an international, peer-reviewed, open access journal covering all aspects of sleep science and sleep medicine, including the neurophysiology and functions of sleep, the genetics of sleep, sleep and society, biological rhythms, dreaming, sleep disorders and therapy, and strategies to optimize healthy sleep.
The manuscript management system is completely online and includes a very quick and fair peer-review system, which is all easy to use. Visit http://www.dovepress.com/testimonials.php to read real quotes from published authors. 